DR. MICHAEL C ANTLE (Orcid ID : 0000-0001-5178-4683)

Article type : Research Report

Reviewers: Gary Pickard, University of Nebraska-Lincoln, USA

Shigenobu Shibata, Waseda University, Japan

Eur J Neurosci

Section: Behavioral Neuroscience

\title{
Chronic BMY7378 treatment alters behavioral circadian rhythms
}

Jhenkruthi Vijaya Shankara ${ }^{1,2}$, Angélique Orr $^{1}$, Richelle Mychasiuk ${ }^{1,2,3}$ and Michael C. Antle $1,2,4, \dagger$

${ }^{1}$ Department of Psychology, ${ }^{2}$ Hotchkiss Brain Institute, ${ }^{3}$ Alberta Children's Hospital Research Institute, and ${ }^{4}$ Department of Physiology and Pharmacology, University of Calgary, Calgary, Alberta, Canada

Short Running Title: $\quad$ Chronic BMY7378 affects the clock

This article has been accepted for publication and undergone full peer review but has not been through the copyediting, typesetting, pagination and proofreading process, which may lead to differences between this version and the Version of Record. Please cite this article as doi: $10.1111 /$ ejn. 13744

This article is protected by copyright. All rights reserved. 
${ }^{\dagger}$ Corresponding Author: Department of Psychology

2500 University Drive NW

Calgary, AB, CANADA

$\mathrm{T} 2 \mathrm{~N} 1 \mathrm{~N} 4$

Phone: $\quad 403-220-2574$

Fax: $\quad 403-282-2849$

Email: $\quad$ antlem@ucalgary.ca

Keywords: $\quad$ Serotonin, photic, phase angle, mixed agonist/antagonist, hamster

\begin{abstract}
The mammalian circadian clock is synchronized to the day:night cycle by light. Serotonin modulates the circadian effects of light, with agonists inhibiting response to light and antagonists enhancing responses to light. A special class of serotonergic compounds, the mixed 5- $\mathrm{HT}_{1 \mathrm{~A}}$ agonist/antagonists, potentiate light-induced phase advances by up to $400 \%$ when administered acutely. In this study, we examine the effects of one of these mixed 5$\mathrm{HT}_{1 \mathrm{~A}}$ agonist/antagonists, BMY7378, when administered chronically. Thirty adult male hamsters were administered either vehicle or BMY7378 via surgically implanted osmotic minipumps over a period of 28 days. In a light:dark cycle, chronic BMY7378 advanced the phase angle of entrainment, prolonged the duration of the active phase, and attenuated the amplitude of the wheel running rhythm during the early night. In constant darkness, chronic treatment with BMY7378 significantly attenuated light-induced phase advances, but had no significant effect on light-induced phase delays. Non-photic phase shifts to daytime
\end{abstract}

This article is protected by copyright. All rights reserved. 


\section{administration of a 5-HT $1 \mathrm{~A} / 7$ agonist were also attenuated by chronic BMY7378}

treatment. qRT-PCR analysis revealed that chronic BMY7378 treatment upregulated mRNA for $5-\mathrm{HT}_{1 \mathrm{~A}}$ and $5-\mathrm{HT}_{1 \mathrm{~B}}$ receptors in the hypothalamus, and downregulated $\mathrm{mRNA}$ for $5-\mathrm{HT}_{1 \mathrm{~A}}$ and monoamine oxidase- $\mathrm{A}$ in the brainstem. These results highlight adaptive changes of serotonin receptors in the brain to chronic treatment with BMY7378, and link such up- and down-regulation to changes in important circadian parameters. Such long-term changes to the circadian system should be considered when patients are treated chronically with drugs that alter serotonergic function.

\section{Introduction}

The mammalian master circadian clock, situated in the suprachiasmatic nucleus (SCN), governs daily rhythms in physiology and behavior (Antle \& Silver, 2005; 2016). These daily internal rhythms are synchronized to the external environment primarily by light input to the retina (Daan \& Pittendrigh, 1976; Pittendrigh \& Daan, 1976; Antle et al., 2009). Light is the predominant cue to which the circadian clock is synchronized and it resets the clock in a phase dependent manner - during the early night, light input delays the phase of the clock, while light input later in the night advances it (Daan \& Pittendrigh, 1976). The serotonergic system plays a role in modulating the magnitude of circadian responses to light (Mistlberger \& Antle, 1998; Rea \& Pickard, 2000). Both serotonin (5-HT) and serotonergic drugs can modulate responses to light (Weber et al., 1998; Morin, 1999; Rea \& Pickard, 2000; Smith et al., 2010; Smith et al., 2014; Smith et al., 2015a).

The SCN receives dense serotonergic input from both the median and dorsal raphe nuclei (Pickard, 1982; Meyer-Bernstein \& Morin, 1996; Pickard \& Rea, 1997a; Yamakawa \& 
Antle, 2010). 5-HT levels in the SCN are highest during periods of high locomotor activity, most notably during the early night for nocturnal rodents (Dudley et al., 1998). In both mice and hamsters, responses to light are generally inhibited by 5-HT agonists (Glass et al., 1994; Rea et al., 1994; Pickard et al., 1996; Pickard \& Rea, 1997b; Weber et al., 1998; Pickard et al., 1999; Rea \& Pickard, 2000; Antle et al., 2003; Smith et al., 2014; Basu et al., 2015) and are enhanced by 5-HT antagonists (Moriya et al., 1998; Byku \& Gannon, 2000; Gannon, 2001; Smart \& Biello, 2001; Takahashi et al., 2002; Gannon, 2003; Gannon \& Millan, 2006; Lall \& Harrington, 2006; Kessler et al., 2008; Sterniczuk et al., 2008; Lungwitz \& Gannon, 2009; Smith et al., 2015a). Of the various 5-HT receptor subtypes, the 5- $\mathrm{HT}_{1 \mathrm{~A}}, 5-\mathrm{HT}_{1 \mathrm{~B}}$ and 5$\mathrm{HT}_{7}$ receptors seem to be important in modulating photic responses (Rea \& Pickard, 2000; Smith et al., 2008; Smith et al., 2015b). 5-HT ${ }_{1 \mathrm{~A}}$ agonists inhibit photic phase shifts in a dose dependent manner when injected systemically or directly into the SCN (Rea et al., 1994; Weber et al., 1998; Antle et al., 2003). They also inhibit firing rates of photically activated SCN cells (Ying \& Rusak, 1994; 1997). This modulation of light-induced responses is mediated by the activation of $5-\mathrm{HT}_{1 \mathrm{~A}}$ and $5-\mathrm{HT}_{7}$ receptors on cells in the $\mathrm{SCN}$ or by inhibition of neurotransmitter release from retinal terminal by activating $5-\mathrm{HT}_{1 \mathrm{~B}}$ receptors (Rea et al., 1994; Pickard et al., 1996; Pickard \& Rea, 1997b; Pickard et al., 1999; Rea \& Pickard, 2000). Conversely, pre-treatment with WAY-100635, a 5- $\mathrm{HT}_{1 \mathrm{~A}}$ antagonist, significantly potentiates light-induced phase delays during the early subjective night (Smart \& Biello, 2001).

A third class of compounds, termed mixed agonist-antagonists, act as agonists at presynaptic somatodendritic $5-\mathrm{HT}_{1 \mathrm{~A}}$ autoreceptors in the raphe nuclei and as antagonists at postsynaptic 5- $\mathrm{HT}_{1 \mathrm{~A}}$ receptors in the SCN (Rydelek-Fitzgerald et al., 1990; Claustre et al., 1991; Gannon, 2003). Four specific compounds have been identified that exhibit this

This article is protected by copyright. All rights reserved. 
pharmacological profile: NAN-190 (Rea et al., 1995; Lall \& Harrington, 2006; Kessler et al., 2008; Sterniczuk et al., 2008; Smith et al., 2010), BMY7378 (Byku \& Gannon, 2000;

Gannon, 2003; Lungwitz \& Gannon, 2009; Smith et al., 2015a), S15535 (Gannon, 2003) and MKC-242 (Moriya et al., 1998; Takahashi et al., 2002). They are of particular interest as they enhance light-induced resetting of the circadian clock in mice and hamsters by up to $400 \%$.

Given the large-amplitude phase shifts, drugs such as these may find application in pharmacotherapy to treat circadian desynchrony associated with rotating shift work, jet lag or chronic sleep disorders. For many airline crews and rotating shift workers, circadian desynchrony can be a chronic situation, which would necessitate chronic treatment, thus, it is important to assess the effects of chronic administration of these compounds. Chronic delivery of some serotonergic drugs can alter various properties of the circadian clock. Chronic delivery of the antidepressant fluoxetine has been reported to shorten freerunning period of mice (Possidente et al., 1996) and to lower activity levels in hamsters (Duncan et al., 2010) although these effects are not universal, as they are not observed in rats (Wollnik, 1992). Chronic treatment with the anxiolytic buspirone lengthens the active phase $(\alpha)$, advances the phase angle of entrainment, and decreases circadian amplitude of hamsters (Smith et al., 2014). Here we use osmotic minipumps to test if chronic BMY7378 alters circadian properties in light-dark cycles and in constant darkness, as well as circadian responses to either phaseshifting light pulses or daytime injections of a 5-HT $\mathrm{TA}_{1 \mathrm{~A}}$ agonist known to cause non-photic phase shifts.

This article is protected by copyright. All rights reserved. 


\section{Methods}

\section{Animals}

A total of $\mathbf{3 0}$ adult male Syrian hamsters were used for this study (Charles River, Kingston, NY, USA). Animals weighed 100-110 g upon arrival in the laboratory. Hamsters were individually housed in polycarbonate cages $(20 \times 45 \times 22 \mathrm{~cm})$ equipped with running wheels (14 cm diameter). Animals were maintained in a 14:10 light:dark (LD) cycle until the start of the experiments. All animals were only used for these studies. Animals had ad libitum access to food (Purina Lab Diet 5001) and water throughout the experiments, and were housed in temperature- $\left(21 \pm 1^{\circ} \mathrm{C}\right)$ and humidity-controlled rooms. Cages were changed approximately every 14 days and at least a week prior to experimental manipulations. All manipulations and husbandry during dark periods were performed using night-vision goggles (General Starlight Company, Richmond Hill, Ontario, Canada). All procedures were approved by the Life and Environmental Sciences Animal Care Committee at the University of Calgary and adhered to the policies of the Canadian Council of Animal Care for the ethical use of animals in research.

\section{General protocol}

Animals were housed in cages equipped with running wheels $(14 \mathrm{~cm}$ diameter, with mesh running surfaces). All animals were initially allowed to entrain to a 14:10 LD cycle (14 hours of 1200 lux and 10 hours of 0 lux). After stable entrainment, osmotic mini pumps were surgically implanted subcutaneously in the region between the hamsters' scapulae. Wheel running was continuously monitored during all studies using magnetic switches mounted to the running wheel and connected to a computer running Clocklab data collection software package (Actimetrics, Wilmette, IL, USA). Actograms were generated using Clocklab analysis software. Ten animals were implanted with pumps containing a solution of the drug 
and ten animals with pumps containing the vehicle solution (saline). Post-surgery all animals were released into their original LD cycles to monitor wheel running activity and examine circadian parameters under light dark cycles for 10-12 days. Following this, animals were placed into constant darkness (DD) for at least 7 days before being exposed to a single phaseshifting light pulse. The osmotic mini pumps only release their solution over a 28 -day period, therefore separate animals were used to examine phase delays $(n=10)$ and phase advances $(n=10)$.

\section{Drugs and Reagents}

The 5- $\mathrm{HT}_{1 \mathrm{~A}}$ receptor mixed agonist/antagonist 8-[2-[4- (2-Methoxyphenyl)-1piperazinyl] ethyl]-8-azaspiro [4.5] decane-7, 9-dione dihydrochloride (BMY7378, Sigma Aldrich, Oakville, ON, USA) was dissolved in physiological saline and administered through osmotic mini pumps. Previously, we had examined the acute effects of this drug (Smith et al., 2015a; Smith et al., 2015c) at a dose of $5 \mathrm{mg} / \mathrm{kg}$. Here we elected to use this same does but spread over the full $24 \mathrm{~h}$ day (i.e., $5 \mathrm{mg} / \mathrm{kg} / \mathrm{day}$ ). To achieve this, pumps were filled with a solution with a concentration of approximately $90 \mathrm{mg} / \mathrm{ml}$. Control pumps were filled with physiological saline (vehicle).

Osmotic mini pumps

All osmotic mini pumps were obtained from Alzet (Alzet Model 2004, DURECT Corporation, Cupertino, CA, USA) and were $2.5 \mathrm{~cm}$ in length and $1 \mathrm{~cm}$ in diameter. All pumps discharged at a rate of approximate $0.25 \mu \mathrm{l} /$ hour for 28 days. All pumps were filled according to manufacturer's instructions. 10 mini pumps in total were filled with BMY7378 and 10 mini pumps were filled with vehicle (physiological saline).

This article is protected by copyright. All rights reserved. 


\section{Surgical procedures}

Animals were entrained to the baseline 14:10 LD cycle before implantation with the osmotic mini pups. On the day of the surgeries osmotic mini pumps were filled with either BMY7378 or physiological saline. Hamsters were anesthetized with sodium pentobarbital $(120 \mathrm{mg} / \mathrm{kg}$, CEVA Santé, France). A small incision was then made in the upper back between the scapulae and a subcutaneous pocket was created by blunt dissection with hemostats. Pumps were implanted subcutaneously and the incision was stapled close. After hamsters fully recovered, they were returned to their home cages with a running wheel and to their previous the LD cycle.

\section{Phase shift manipulations}

For the phase shifting experiments animals were first allowed to free run in DD for 7-9 days. They were then subjected to light pulses (15 min, 40 lux) either 6 hours after activity onset (circadian time (CT)18; with CT12 being defined by convention as the time of activity onset for nocturnal animals) or an hour after activity onset (CT13). These phases elicit maximal phase advances and phase delays respectively, in Syrian hamsters (Daan \& Pittendrigh, 1976). Because the mini pumps only discharged at a constant rate for a period of 28 days, two sets of animals were used to separately test phase shifts at each of the time points. Ten animals in total were used to test for phase advances -5 animals were implanted with BMY7378 filled mini pumps and 5 with saline filled mini pumps. Nine animals were used to test for phase delays -5 animals were implanted with BMY7378 mini pumps and 5 with saline, however one control animal was excluded from analysis due to illness. After exposure to the light pulses animals were returned to $\mathrm{DD}$ and allowed to free run.

This article is protected by copyright. All rights reserved. 
A further set of animals ( $n=5$ with BMY7378 filled mini pumps and $n=5$ with saline filled mini pumps) were used to explore non-photic phase shifts to the serotonin 1A/7 agonists 8-hydroxy-2-(di-n-propylamino) (8-OH-DPAT, 5mg/kg in saline, Sigma,

\section{St. Louis, MO). Injections occurred on day 8 of DD at CT8.} $q R T-P C R$

On the last day that the osmotic minipumps would deliver drug, animals received an overdose of sodium pentobarbital at either CT0 or CT12. Brains were extracted and flash frozen on dry ice. Tissue from the prefrontal cortex, hypothalamus and brainstem (midbrain through hindbrain less the cerebellum, thus containing the major raphe nuclei) was removed and total RNA was extracted using the Allprep RNA/DNA Mini Kit following the manufacturer's protocols (Qiagen, Germany). Quality and quantity of RNA was ascertained with a Nanodrop 2000 (Thermo Fisher Scientific, Waltham, MA). To synthesize cDNA, $2 \mu \mathrm{g}$ of RNA per sample was reverse transcribed using the qScript ${ }^{\mathrm{TM}}$ XLT cDNA Supermix (Quanta Biosciences, Gaithersburg, MD), using the manufacturer's protocols. Primers for the 5- $\mathrm{HT}_{1 \mathrm{~A}}$ receptor, the 5- $\mathrm{HT}_{1 \mathrm{~B}}$ receptor, and monoamine oxidase $\mathrm{A}$ (MAO-A), were manually designed by an in-house technician and purchased from IDT (Coalville, IA). Primer sequences were as follows: MAO-A, forward: gccaggaacggaaatttgtagg, reverse: ttggtttctctcaggtggaagc yielding a 215 base pair (bp) fragment; 5-HT ${ }_{1 \mathrm{~A}}$ : forward: atatagaaagcgccgaaagtgg, reverse: actatgtgaacaaaaggacgec yielding a 164 bp fragment, 5$\mathrm{HT}_{1 \mathrm{~B}}$ : forward: tacgtgaaccaagtcaaagtgc, reverse: accagggagatgatgaagaagg yielding a 218 bp fragment. Each sample was run in duplicate, using $10 \mathrm{ng}$ of cDNA, $0.5 \mu \mathrm{M}$ of the forward and reverse primers, and 1x SYBRGreenFastMix. qRT-PCR was performed and analyzed with the CFX Connect Real-Time PCR detection system. (Bio-Rad, Hercules, CA). To

This article is protected by copyright. All rights reserved. 
determine the relative gene expression, each sample was normalized against two

housekeeping genes: Ywhaz and $C y c A$ (Bonefeld et al., 2008) using the $2^{-\Delta \Delta \mathrm{Ct}}$ method as described by Pfaffl (2001). Primer sequences for the housekeeping genes were as follows: $C y c A$, forward: agcactggggagaaaggatt, reverse: agccactcagtcttggcagt yielding a $248 b p$ fragment; Ywhaz: forward: ttgagcagaagacggaaggt, reverse: gaagcattggggatcaagaa yielding a 136 bp fragment.

Data analysis

Data analysis was conducted with the Clocklab Analysis package (Actimetrics, Wilmette, IL, USA). Phase angle was assessed over the 5 days prior to surgery and the 5 days following surgery. Clocklab's activity onset identification tool determined onset times, which were subtracted from the time of lights-off. Positive values indicated activity onsets that preceded lights-off. Phase angle results were analyzed with a 2 (baseline vs treatment) x 2 (BMY7378 vs saline) factorial Analysis of Variance (ANOVA). Activity duration (alpha) was calculated by having Clocklab identify the onsets and offsets on each day. The difference between these was calculated, and the average was calculated over the days being analyzed (last 6 days of baseline, and first 5 days after surgery). Alpha was analyzed using a 2x2 mixed factorial ANOVA. Activity levels were assessed for the same days, and were averaged together to yield average waveforms. Activity counts were averaged into four separate blocks: over the day, and over the 3 equal blocks during the night. Two 2 (baseline vs treatment) x 4 (block) factorial ANOVAs were used to analyze the activity level results. Saline and BMY animals were analyzed separately in this case, and planned contrasts between activity levels in each block between baseline and treatment were performed. Phase shifts to light pulses and 8-OH-DPAT were calculated using the standard Clocklab routine as described 
previously (Sterniczuk et al., 2008). Briefly, regression lines were fitted for activity onset a week before the light pulse and for days 3-10 after the light pulse. The first two days after the light pulse were not analyzed to allow for transient effects. The post manipulation regression line is then extrapolated back to the day after the light pulse. The horizontal difference between the two regression lines represented the phase shift. Phase shifts were analyzed using independent samples t-tests. qRT-PCR results from the hypothalamus and prefrontal cortex were analyzed with separate 2 (treatment: saline vs BMY7378) x 2 (phase: CT0 vs CT12) two-way ANOVAs. Each gene and brain area was analyzed with separate ANOVAs. Due to experimenter error, brainstem samples from CT0 were lost, so brainstem samples were only analyzed at CT12. Separate independent samples t-tests were run for each gene comparing saline to BMY7378. As BMY7378 acts as an agonist at 5-HT1A autoreceptors in the brainstem raphe nuclei (Sharp \& Hjorth, 1990), we hypothesized that this would lead to downregulation of the 5-HT1A receptors here, and therefore used a one-tailed t-test for this gene, while using two tailed tests for the other genes. For ANOVAs, all significant main effects and interactions were followed-up using Student-Newman-Keuls post hoc tests.

\section{Results}

Animals treated with BMY7378 exhibited a rapid change to their circadian activity patterns. The overall waveform of wheel-running activity exhibited a decrease in amplitude. This was significant during both the early- and mid-night (significant treatment $\mathbf{x}$ block interaction, $F_{(3,42)}=7.584 p<0.001$, Figure 1), with a small but significant increase in the late-night block. Vehicle treated animals also exhibited a significant decrease in the 
amplitude of their waveforms after surgery (main effect of treatment, $\boldsymbol{F}_{(\mathbf{1}, 30)}=\mathbf{5 . 2 1 9}$ $p=0.045)$. While the there was no significant interaction between treatment and time of day $\left(F_{(3,30)}=1.754 p=0.177\right)$ the planned comparisons revealed that the mid-night block was the only time when activity levels differed significantly $(p=0.006)$ between baseline and treatment in the vehicle treated animals. The actograms and waveforms also suggested a change in phase angle of entrainment. Treatment with BMY7378 significantly advances the activity onset relative to saline post-surgery as well as relative to the baseline phase angle prior to surgery in the same animals (significant pump type $\mathrm{x}$ treatment block interaction, $F_{(2,48)}=13.552, p<0.001$, Figure 2). This difference was most striking in the days immediately following implantation of the pump ( $p<0.001$ both vs. saline and vs. baseline). While the phase angle of BMY7378 treated animals was significantly smaller by days 10-14 after surgery $(p<0.001)$, it was still significantly greater than that of both the saline-treated animals $(p=0.016)$ and their own baseline phase angle $(p<0.001)$. This advance in phase angle likely contributed to a significant expansion of the active phase ( $\alpha$ ) from 5.68 $\pm \mathbf{0 . 2 4 h}$ during baseline to $\mathbf{7 . 1 7} \pm \mathbf{0 . 3 4 h}$ during treatment with BMY7378 (significant treatment $\mathrm{x}$ block interaction, $\boldsymbol{F}_{(\mathbf{1 , 2 4})}=\mathbf{1 1 . 5 9 0} \boldsymbol{p = \mathbf { 0 . 0 0 2 }}$ ). The $\alpha$ of saline treated animals did not differ between baseline $(\mathbf{6 . 3 7} \pm \mathbf{0 . 3 6 h})$ and treatment $(\mathbf{6 . 2 3} \pm \mathbf{0 . 4 8 h})$.

While activity levels dropped after pump implantation (Saline baseline $\mathbf{9 7 7 5 . 1 \pm 6 9 7}$

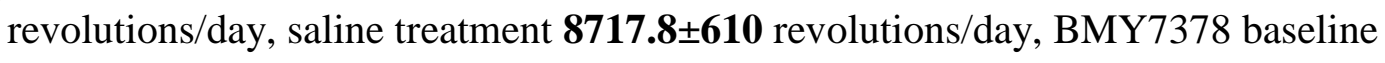
9799.2 $\pm \mathbf{4 8 2}$ revolutions/day, BMY7378 treatment $\mathbf{9 3 5 5 . 0 \pm 5 0 8}$ revolutions/day; main effect of treatment block $\left.\boldsymbol{F}_{(\mathbf{1}, \mathbf{2 4})}=\mathbf{7 . 5 8 8} \boldsymbol{p}=\mathbf{0 . 0 1 1}\right)$, this did not differ between saline and BMY7378treated animals (interaction $\boldsymbol{F}_{(\mathbf{1}, 24)}=\mathbf{1 . 2 6 5} \boldsymbol{p}=\mathbf{0 . 2 7 2}$ ). There was also no difference in free-

This article is protected by copyright. All rights reserved. 
running period between the saline and BMY7378 treated animals when they were put into DD (Saline: $24.09 \pm 0.01 \mathrm{~h}, \mathrm{BMY} 7378: 24.10 \pm 0.02 \mathrm{~h}, t_{(17)}=0.42, p=0.68$ ).

Animals pretreated with BMY7378 had significantly smaller phase advances to a late night light pulse $(\mathbf{0 . 3 4} \pm \mathbf{0 . 0 7} \mathbf{h})$ compared to those treated with saline $\left(\mathbf{1 . 0 5} \pm \mathbf{0 . 0 8 h}, t_{(8)}=6.706\right.$, $p=0.002$, Figure 3 ). BMY7378 pretreatment did not significantly change the magnitude of phase delays to early night light pulses (saline: $-\mathbf{0 . 7 0 \pm 0 . 2 8 h , ~ B M Y 7 3 7 8 : ~}-\mathbf{0 . 9 5} \pm 0.12 \mathrm{~h}$, $\left.t_{(7)}=0.8873, p=0.40\right)$. Non-photic phase shifts to CT8 injections of 8-OH-DPAT $(0.384 \pm 0.11 \mathrm{~h}$ in animals with saline filled pumps) were significantly attenuated in animals with BMY7378-filled pumps $\left(0.042 \pm 0.06 \mathrm{~h}, t_{(8)}=2.64, p=0.030\right.$, Figure 4).

Chronic treatment with BMY7378 altered gene expression of various serotoninrelated genes. BMY7378 increased levels of $5-\mathrm{HT}_{1 \mathrm{~A}}$ receptor mRNA in the hypothalamus $\left(F_{(1,15)}=9.088, p=0.009\right.$, Figure 5) but decreased it in the brainstem $\left(t_{(7)}=2.18, p=0.033\right.$, Figure 6). There was no significant change in the levels of $5-\mathrm{HT}_{1 \mathrm{~A}}$ receptor mRNA in the prefrontal cortex $\left(F_{(1,15)}=0.984, p=0.337\right)$. BMY7378 increased levels of $5-\mathrm{HT}_{1 \mathrm{~B}}$ receptor mRNA in the hypothalamus $\left(F_{(1,15)}=11.590, p=0.004\right.$, Figure 5$)$. While levels of $5-\mathrm{HT}_{1 \mathrm{~B}}$ receptor mRNA appeared lower in the prefrontal cortex in BMY7378-treated animals, this main effect was not significant $\left(F_{(1,13)}=4.445, p=0.055\right.$, Figure 5). There was no significant change in the levels of $5-\mathrm{HT}_{1 \mathrm{~B}}$ receptor $\mathrm{mRNA}$ in the brainstem $\left(t_{(7)}=\mathbf{0 . 1 0 6}\right.$, $p=0.918$, Figure 6). BMY7378 decreased levels of monoamine oxidase A mRNA in the prefrontal cortex $\left(F_{(1,15)}=9.714, p=0.007\right.$, Figure 5). While levels of monoamine oxidase A mRNA appeared lower in the hypothalamus in BMY7378-treated animals, this main effect was not significant $\left(F_{(1,15)}=4.432, p=0.053\right.$, Figure 5). Levels of monoamine oxidase A mRNA were significantly lower in the brainstem of BMY7378-treated animals $\left(t_{(7)}=\right.$ 
3.00, $p=0.01$, Figure 6). The only significant time of day main effect detected was for monoamine oxidase A mRNA levels in the prefrontal cortex $\left(F_{(1,13)}=19.127, p<0.001\right.$, Figure 5) with higher levels at CT0. None of the treatment $x$ phase interactions were significant (all $p>0.2)$.

\section{Discussion}

The present study examined the effects of chronic BMY7378 treatment on circadian behavior in male hamsters. BMY7378 is a $5-\mathrm{HT}_{1 \mathrm{~A}}$ mixed agonist/antagonist that potentiates phase advances to light when acutely administered systemically (Byku \& Gannon, 2000; Gannon, 2003; Smith et al., 2010; Smith et al., 2015a). We found that chronic BMY7378 administration significantly advanced the phase angle of entrainment, and decreased the amplitude of activity in the early part of the night. Chronic BMY7378 also attenuates phase advances to late-night light pulses and daytime injections of 8-OH-DPAT, but had no effect on phase delays to early-night light pulses. Chronic BMY7378 had no effect on free-running period or overall activity levels. Chronic BMY7378 also leads to upregulation of 5-HT $\mathrm{HA}_{1 \mathrm{~A}}$ and $5-\mathrm{HT}_{1 \mathrm{~B}}$ mRNA levels in the hypothalamus, and downregulation of mRNA for both the $5-\mathrm{HT}_{1 \mathrm{~A}}$ receptor and MAO-A in the brainstem.

The mechanism by which acute treatment with BMY7378 and other 5-HT $\mathrm{HA}_{1 \mathrm{~A}}$ mixed agonist/antagonists enhances advances to light are complex. The 5- $\mathrm{HT}_{1 \mathrm{~A}}$ receptor plays a key role in these responses (Smith et al., 2010). BMY7378 appears to exert its proximate effects at the raphe nuclei (Smith et al., 2015a) where it will decrease serotonin output by activating 5- $\mathrm{HT}_{1 \mathrm{~A}}$ autoreceptors. This would lead to disinhibition of both retinal terminals in the SCN and of SCN cells themselves. This would lead to both enhanced neurotransmitter 
release in response to a light pulse, and enhanced post-synaptic responses to these inputs.

\section{Supporting this latter hypothesis, we have previously demonstrated these mixed} agonist/antagonist drugs enhance phase shifts to intraSCN injections of NMDA and gastrin-releasing peptide (Sterniczuk et al., 2008). Additionally, serotonergic input to the SCN is not necessary for BMY7378 to enhance photic phase shifts (Smith et al., 2015a) suggesting that other input pathways are involved, such as from the intergeniculate leaflet. In contrast to the mechanism of action for acute treatments, the mechanism of action for the responses observed here to chronic treatment may simply involve upregulation and downregulation of genes involved in regulating the activity of serotonin. Consistent with its role as an agonist at 5- $\mathrm{HT}_{1 \mathrm{~A}}$ autoreceptors, BMY7378 leads to a downregulation of the mRNA for the 5-HT $\mathrm{HA}_{1 \mathrm{~A}}$ autoreceptor as well as for MAO-A. If these changes are mirrored in the levels of their corresponding proteins, then these changes could enhance serotonergic tone. Additionally, BMY7378 also acts as an antagonist for postsynaptic 5-HT AA $_{\text {A }}$ receptors. Chronic antagonism would lead to upregulation of the receptors, as was observed for levels of 5-HT $\mathrm{H}_{1 \mathrm{~A}}$ mRNA in the hypothalamus. So, while in the acute situation the circadian system would have very low serotonergic activity with the raphe being silenced and the post-synaptic $5-\mathrm{HT}_{1 \mathrm{~A}}$ receptors being blocked, in the chronic situation the opposite situation emerges, with the raphe being desensitized to negative feedback and post synaptic serotonin targets being more sensitive.

The observed changes in phase advances are consistent with a system where serotonin release is enhanced, owing to downregulation of 5- $\mathrm{HT}_{1 \mathrm{~A}}$ receptors and $\mathrm{MAO}-\mathrm{A}$ in the brainstem, and where the SCN has enhanced sensitivity to serotonin, owing to upregulation of both 5- $\mathrm{HT}_{1 \mathrm{~A}}$ and 5- $\mathrm{HT}_{1 \mathrm{~B}}$ receptors in the hypothalamus. During the late night, when light

This article is protected by copyright. All rights reserved. 
exposure can cause phase advances, serotonin levels in the SCN are at or just above their daily mean level (Dudley et al., 1998). Given serotonin's inhibitory role over photic responses of the circadian system (Rea \& Pickard, 2000), any enhancement of the release of serotonin or responses to serotonin should inhibit phase shifts to light. During the early night when light produces phase shifts, serotonin is at its highest levels in the SCN (Dudley et al., 1998), thus any further enhancement in serotonin release is unlikely to exhibit any inhibition of shifts to phase-delaying light, as these phases shifts in unmanipulated animals are already occurring under maximal serotonergic inhibition.

Our study also found that animals administered BMY7378 chronically had a significantly more positive phase angle compared to vehicle treated animals - drug treated animals started their activity on average half an hour before light onset compared to vehicle treated animals. There was also a corresponding expansion of the duration of their active phase. Similar changes have been reported with neurotoxic lesions of the median raphe (Meyer-Bernstein et al., 1997). Given that the changes in phase angle were most prominent in the initial days following implantation of the minipumps, this could represent initial activation of 5- $\mathrm{HT}_{1 \mathrm{~A}}$ autoreceptors, leading to diminished raphe output, yielding similar behavioral results to those observed when serotonergic input to the circadian system is eliminated with neurotoxic lesions (Meyer-Bernstein et al., 1997). Alternatively, the advanced phase angle might be due to initial enhanced advances to dawn light on the first day following implantation of minipump before up- and down-regulation of receptors occurred. A third option could be a change in the shape of the phase response curve to light, which would alter the phase angle of entrainment. This last option is intriguing, given that phase advances to light were eliminated in this study. However, loss of the advance region should then bias the 
phase response curve to delays, which should lead to more negative, rather than more positive, phase angle. However, given that the changes to phase angle diminished over the study, it is unlikely that the serotonergic system was in a similar state between when the phase angle changes (first few days) and phase shift changes were observed (3 weeks into chronic treatment). The final option for explaining the phase angle is a shortened circadian period, but there were no changes in period observed in these animals when placed into constant darkness, making this unlikely as well, although again period and phase angle we assessed at different timepoints in the study, and the serotonergic system may not have been equivalent between them.

Activity levels overall did not change during treatment with BMY7378. The amplitude of activity in the early part of the night was decreased during BMY7378 treatment, but the advanced phase angle of entrainment may have redistributed activity into the daytime. Wheel running behavior can be homeostatically regulated (Mueller et al., 1999), thus the decrease in activity observed in the early night may simply be a homeostatic response to the activity that occurred prior to dark-onset during BMY7378 treatment.

Serotonin and the 5- $\mathrm{HT}_{1 \mathrm{~A}}$ and 5- $\mathrm{HT}_{7}$ receptors have also been implicated in non-photic phase shifting to exercise and arousal manipulations (Mistlberger et al., 2000; Antle et al., 2003; Gardani \& Biello, 2008; Smith et al., 2008; Yamakawa \& Antle, 2010; Smith et al., 2015b), however some studies have questioned this (Antle et al., 1998; Meyer-Bernstein \& Morin, 1998; Antle et al., 2000). While numerous studies have reported non-photic phase shifts to administration of the 5-HT $1 \mathrm{~A} / 7$ agonist 8-OH-DPAT (Tominaga et al., 1992; Mintz et al., 1997; Antle et al., 2003), the site of action of this drug has debated. In vitro studies have suggested a role at the SCN itself (Prosser et al., 1993; Prosser, 2003), while 
in vivo microinjections to the $\mathrm{SCN}$ of hamsters were unable to mimic this response (Mintz et al., 1997; Antle et al., 2003). Microinjections of 8-OH-DPAT to the dorsal and median raphe did elicit non-photic phase shifts (Mintz et al., 1997). Given the upregulation of serotonin receptors in the $\mathrm{SCN}$ and downregulation of these receptors in the brainstem, the current study allows us to discriminate between these two hypotheses. If systemic 8-OH-DPAT acts primarily at the $\mathrm{SCN}$, the upregulation of receptors there would be expected to lead to enhanced phase shifts to 8-OH-DPAT. However, as phase shifts were significantly attenuated, this observation is more in line with the hypothesis that it act primarily at the raphe, where the observed downregulation of receptors would predict a smaller response. A similar approach could be used in the future to explore serotonin's role in other non-photic shifts, such as those to arousal, sleep deprivation or exercise (Bobrzynska \& Mrosovsky, 1998; Antle \& Mistlberger, 2000; Yamakawa et al., 2016). With the receptor changes observed here, animals treated with chronic BMY7378 should have a system in which both release of and responses to serotonin are enhanced. If serotonin plays a role in non-photic shifts, then phase shifts may be enhanced in animals chronically treated with BMY7378.

In conclusion, this study shows that while BMY7378 can greatly enhance responses to light when used acutely, chronic application of BMY7378 is quite different in that phase advances to light are completely absent. Chronic BMY treatment also leads to changes in the amplitude of the wheel running rhythm, the duration of the active phase, and the phase angle of entrainment. This chronic treatment with BMY7378 also leads to up- and down-regulation of certain components of the serotonergic system consistent with BMY7378's role as a mixed agonist-antagonist for the 5- $\mathrm{HT}_{1 \mathrm{~A}}$ receptor. These findings not only highlight the role of

This article is protected by copyright. All rights reserved. 
serotonin in the circadian system, but also its adaptive responses to chronic stimulation. These changes are important to consider if serotonergic compounds are used in a chronic fashion to treat various sleep and circadian disorders.

\section{Acknowledgements}

This work was supported by a Discovery Grant from the Natural Sciences and Engineering Research Council (NSERC) of Canada to MCA, and by Startup fund from the Alberta Children's Hospital Research Foundation to RM.

\section{Competing Interests}

The authors declare no conflicts of interest.

\section{Author Contributions}

JVS - Conducted the experiments, analyzed the data, and wrote the manuscript. AO Conducted the experiments and analyzed the data. $\mathrm{RM}$ - Conducted the experiments, analyzed the data, and wrote the manuscript. MCA - Designed and supervised the experiments, analyzed the data, and wrote the manuscript.

\section{Data Accessibility}

Actograms and spreadsheets are available through the University of Calgary's

Dataverse platform:

https://dataverse.scholarsportal.info/dataset.xhtml?persistentId=doi:10.5683/SP/6BRBIR

This article is protected by copyright. All rights reserved. 


\section{References}

Antle, M.C., Glass, J.D. \& Mistlberger, R.E. (2000) 5-HT $(1 \mathrm{~A})$ autoreceptor antagonist-induced 5-HT release in the hamster suprachiasmatic nuclei: effects on circadian clock resetting. Neurosci Lett, 282, 97-100.

Antle, M.C., Marchant, E.G., Niel, L. \& Mistlberger, R.E. (1998) Serotonin antagonists do not attenuate activity-induced phase shifts of circadian rhythms in the Syrian hamster. Brain Res, 813, 139-149.

Antle, M.C. \& Mistlberger, R.E. (2000) Circadian clock resetting by sleep deprivation without exercise in the Syrian hamster. J Neurosci, 20, 9326-9332.

Antle, M.C., Ogilvie, M.D., Pickard, G.E. \& Mistlberger, R.E. (2003) Response of the mouse circadian system to serotonin 1A/2/7 agonists in vivo: surprisingly little. $J$ Biol Rhythms, 18, 145-158.

Antle, M.C. \& Silver, R. (2005) Orchestrating time: arrangements of the brain circadian clock. Trends Neurosci, 28, 145-151.

Antle, M.C. \& Silver, R. (2016) Circadian Insights into Motivated Behavior. Curr Top Behav Neurosci, 27, 137-169.

Antle, M.C., Smith, V.M., Sterniczuk, R., Yamakawa, G.R. \& Rakai, B.D. (2009) Physiological responses of the circadian clock to acute light exposure at night. Rev Endocr Metab Disord, 10, 279-291.

Basu, P., Ie, N., Wensel, A.L., Baskerville, J.D., Smith, V.M. \& Antle, M.C. (2015) Triptans attenuate circadian responses to light. Eur J Neurosci, 42, 2489-2495.

This article is protected by copyright. All rights reserved. 
Bobrzynska, K.J. \& Mrosovsky, N. (1998) Phase shifting by novelty-induced running: activity dose-response curves at different circadian times. Journal of comparative physiology. A, Sensory, neural, and behavioral physiology, 182, 251-258.

Bonefeld, B.E., Elfving, B. \& Wegener, G. (2008) Reference genes for normalization: a study of rat brain tissue. Synapse, 62, 302-309.

Byku, M. \& Gannon, R.L. (2000) Effects of the 5HT1A agonist/antagonist BMY 7378 on light-induced phase advances in hamster circadian activity rhythms during aging. $J$ Biol Rhythms, 15, 300-305.

Claustre, Y., Rouquier, L., Serrano, A., Benavides, J. \& Scatton, B. (1991) Effect of the putative 5-HT1A receptor antagonist NAN-190 on rat brain serotonergic transmission. Eur J Pharmacol, 204, 71-77.

Daan, S. \& Pittendrigh, C.S. (1976) A functional analysis of circadian pacemakers in nocturnal rodents. II. The variability of phase response curves. J Comp Physiol, 106, 253-266.

Dudley, T.E., DiNardo, L.A. \& Glass, J.D. (1998) Endogenous regulation of serotonin release in the hamster suprachiasmatic nucleus. J Neurosci, 18, 5045-5052.

Duncan, M.J., Hester, J.M., Hopper, J.A. \& Franklin, K.M. (2010) The effects of aging and chronic fluoxetine treatment on circadian rhythms and suprachiasmatic nucleus expression of neuropeptide genes and 5-HT1B receptors. Eur J Neurosci, 31, 16461654.

Gannon, R.L. (2001) $5 \mathrm{HT}_{7}$ receptors in the rodent suprachiasmatic nucleus. J Biol Rhythms, 16, 19-24.

This article is protected by copyright. All rights reserved. 
Gannon, R.L. (2003) Serotonergic serotonin (1A) mixed agonists/antagonists elicit largemagnitude phase shifts in hamster circadian wheel-running rhythms. Neuroscience, 119, 567-576.

Gannon, R.L. \& Millan, M.J. (2006) Serotonin1A autoreceptor activation by S 15535 enhances circadian activity rhythms in hamsters: evaluation of potential interactions with serotonin2A and serotonin2C receptors. Neuroscience, 137, 287-299.

Gardani, M. \& Biello, S.M. (2008) The effects of photic and nonphotic stimuli in the 5-HT7 receptor knockout mouse. Neuroscience, 152, 245-253.

Glass, J.D., Selim, M. \& Rea, M.A. (1994) Modulation of light-induced C-Fos expression in the suprachiasmatic nuclei by 5-HT1A receptor agonists. Brain Res, 638, 235-242.

Kessler, E.J., Sprouse, J. \& Harrington, M.E. (2008) NAN-190 potentiates the circadian response to light and speeds re-entrainment to advanced light cycles. Neuroscience, 154, 1187-1194.

Lall, G.S. \& Harrington, M.E. (2006) Potentiation of the resetting effects of light on circadian rhythms of hamsters using serotonin and neuropeptide Y receptor antagonists. Neuroscience, 141, 1545-1552.

Lungwitz, E. \& Gannon, R.L. (2009) Serotonin1A-mediated amplification of light-induced phase advances of circadian rhythms in the Syrian hamster: post-light effects. Brain Res, 1250, 157-163.

Meyer-Bernstein, E.L., Blanchard, J.H. \& Morin, L.P. (1997) The serotonergic projection from the median raphe nucleus to the suprachiasmatic nucleus modulates activity phase onset, but not other circadian rhythm parameters. Brain Res, 755, 112-120.

This article is protected by copyright. All rights reserved. 
Meyer-Bernstein, E.L. \& Morin, L.P. (1996) Differential serotonergic innervation of the suprachiasmatic nucleus and the intergeniculate leaflet and its role in circadian rhythm modulation. J Neurosci, 16, 2097-2111.

Meyer-Bernstein, E.L. \& Morin, L.P. (1998) Destruction of serotonergic neurons in the median raphe nucleus blocks circadian rhythm phase shifts to triazolam but not to novel wheel access. J Biol Rhythms, 13, 494-505.

Mintz, E.M., Gillespie, C.F., Marvel, C.L., Huhman, K.L. \& Albers, H.E. (1997) Serotonergic regulation of circadian rhythms in Syrian hamsters. Neuroscience, 79, 563-569.

Mistlberger, R.E. \& Antle, M.C. (1998) Behavioral inhibition of light-induced circadian phase resetting is phase and serotonin dependent. Brain Res, 786, 31-38.

Mistlberger, R.E., Antle, M.C., Glass, J.D. \& Miller, J.D. (2000) Behavioral and serotonergic regulation of circadian rhythms. Biol Rhythm Res, 31, 240-283.

Morin, L.P. (1999) Serotonin and the regulation of mammalian circadian rhythmicity. Ann Med, 31, 12-33.

Moriya, T., Yoshinobu, Y., Ikeda, M., Yokota, S., Akiyama, M. \& Shibata, S. (1998) Potentiating action of MKC-242, a selective 5-HT1A receptor agonist, on the photic entrainment of the circadian activity rhythm in hamsters. Br J Pharmacol, 125, 12811287.

Mueller, D.T., Herman, G. \& Eikelboom, R. (1999) Effects of short- and long-term wheel deprivation on running. Physiol Behav, 66, 101-107.

Pfaffl, M.W. (2001) A new mathematical model for relative quantification in real-time RTPCR. Nucleic Acids Res, 29, e45.

This article is protected by copyright. All rights reserved. 
Pickard, G.E. (1982) The afferent connections of the suprachiasmatic nucleus of the golden hamster with emphasis on the retinohypothalamic projection. J Comp Neurol, 211, 6583.

Pickard, G.E. \& Rea, M.A. (1997a) Serotonergic innervation of the hypothalamic suprachiasmatic nucleus and photic regulation of circadian rhythms. Biol Cell, 89, 513-523.

Pickard, G.E. \& Rea, M.A. (1997b) TFMPP, a 5HT 1 1B receptor agonist, inhibits light-induced phase shifts of the circadian activity rhythm and c-Fos expression in the mouse suprachiasmatic nucleus. Neurosci Lett, 231, 95-98.

Pickard, G.E., Smith, B.N., Belenky, M., Rea, M.A., Dudek, F.E. \& Sollars, P.J. (1999) 5$\mathrm{HT}_{1 \mathrm{~B}}$ receptor-mediated presynaptic inhibition of retinal input to the suprachiasmatic nucleus. J Neurosci, 19, 4034-4045.

Pickard, G.E., Weber, E.T., Scott, P.A., Riberdy, A.F. \& Rea, M.A. (1996) $5 \mathrm{HT}_{1 \mathrm{~B}}$ receptor agonists inhibit light-induced phase shifts of behavioral circadian rhythms and expression of the immediate-early gene c-fos in the suprachiasmatic nucleus. $J$ Neurosci, 16, 8208-8220.

Pittendrigh, C.S. \& Daan, S. (1976) A functional analysis of circadian pacemakers in nocturnal rodents. IV. Entrainment: Pacemaker as clock. J Comp Physiol, 106, 291331.

Possidente, B., Lumia, A.R., McGinnis, M.Y., Rapp, M. \& McEldowney, S. (1996) Effects of fluoxetine and olfactory bulbectomy on mouse circadian activity rhythms. Brain Res, 713, 108-113.

This article is protected by copyright. All rights reserved. 
Prosser, R.A. (2003) Serotonin phase-shifts the mouse suprachiasmatic circadian clock in vitro. Brain Res, 966, 110-115.

Prosser, R.A., Dean, R.R., Edgar, D.M., Heller, H.C. \& Miller, J.D. (1993) Serotonin and the mammalian circadian system: I. In vitro phase shifts by serotonergic agonists and antagonists. J Biol Rhythms, 8, 1-16.

Rea, M.A., Barrera, J., Glass, J.D. \& Gannon, R.L. (1995) Serotonergic potentiation of photic phase shifts of the circadian activity rhythm. Neuroreport, 6, 1417-1420.

Rea, M.A., Glass, J.D. \& Colwell, C.S. (1994) Serotonin modulates photic responses in the hamster suprachiasmatic nuclei. J Neurosci, 14, 3635-3642.

Rea, M.A. \& Pickard, G.E. (2000) Serotonergic modulation of photic entrainment in the Syrian hamster. Biol Rhythm Res, 31, 284-314.

Rydelek-Fitzgerald, L., Teitler, M., Fletcher, P.W., Ismaiel, A.M. \& Glennon, R.A. (1990) NAN-190: agonist and antagonist interactions with brain 5-HT1A receptors. Brain Res, 532, 191-196.

Sharp, T. \& Hjorth, S. (1990) Application of brain microdialysis to study the pharmacology of the 5-HT1A autoreceptor. Journal of neuroscience methods, 34, 83-90.

Smart, C.M. \& Biello, S.M. (2001) WAY-100635, a specific 5-HT $1 \mathrm{~A}$ antagonist, can increase the responsiveness of the mammalian circadian pacemaker to photic stimuli. Neurosci Lett, 305, 33-36.

Smith, V.M., Hagel, K. \& Antle, M.C. (2010) Serotonergic potentiation of photic phase shifts: examination of receptor contributions and early biochemical/molecular events. Neuroscience, 165, 16-27.

This article is protected by copyright. All rights reserved. 
Smith, V.M., Iannattone, S., Achal, S., Jeffers, R.T. \& Antle, M.C. (2014) The serotonergic anxiolytic buspirone attenuates circadian responses to light. Eur J Neurosci, 40, 35123525.

Smith, V.M., Jeffers, R.T. \& Antle, M.C. (2015a) Serotonergic enhancement of circadian responses to light: role of the raphe and intergeniculate leaflet. Eur J Neurosci, $\mathbf{4 2}$, 2805-2817.

Smith, V.M., Jeffers, R.T., McAllister, B.B., Basu, P., Dyck, R.H. \& Antle, M.C. (2015b) Effects of lighting condition on circadian behavior in 5-HT1A receptor knockout mice. Physiol Behav, 139, 136-144.

Smith, V.M., Jeffers, R.T., Wu, C., Vijaya Shankara, J. \& Antle, M.C. (2015c) Temporal changes of light-induced proteins in the $\mathrm{SCN}$ following treatment with the serotonin mixed agonist/antagonist BMY7378. Exp Brain Res, 233, 2723-2731.

Smith, V.M., Sterniczuk, R., Phillips, C.I. \& Antle, M.C. (2008) Altered photic and nonphotic phase shifts in 5-HT(1A) receptor knockout mice. Neuroscience, 157, 513-523.

Sterniczuk, R., Stepkowski, A., Jones, M. \& Antle, M.C. (2008) Enhancement of photic shifts with the 5-HT $1 \mathrm{~A}$ mixed agonist/antagonist NAN-190: Intra-suprachiasmatic nucleus pathway. Neuroscience, 153, 571-580.

Takahashi, S., Yoshinobu, Y., Aida, R., Shimomura, H., Akiyama, M., Moriya, T. \& Shibata, S. (2002) Extended action of MKC-242, a selective 5-HT(1A) receptor agonist, on light-induced Per gene expression in the suprachiasmatic nucleus in mice. J Neurosci Res, 68, 470-478.

This article is protected by copyright. All rights reserved. 
Tominaga, K., Shibata, S., Ueki, S. \& Watanabe, S. (1992) Effects of 5-HT ${ }_{1 \mathrm{~A}}$ receptor agonists on the circadian rhythm of wheel- running activity in hamsters. Eur $J$ Pharmacol, 214, 79-84.

Weber, E.T., Gannon, R.L. \& Rea, M.A. (1998) Local administration of serotonin agonists blocks light-induced phase advances of the circadian activity rhythm in the hamster. $J$ Biol Rhythms, 13, 209-218.

Wollnik, F. (1992) Effects of chronic administration and withdrawal of antidepressant agents on circadian activity rhythms in rats. Pharmacol Biochem Behav, 43, 549-561.

Yamakawa, G.R. \& Antle, M.C. (2010) Phenotype and function of raphe projections to the suprachiasmatic nucleus. Eur J Neurosci, 31, 1974-1983.

Yamakawa, G.R., Basu, P., Cortese, F., MacDonnell, J., Whalley, D., Smith, V.M. \& Antle, M.C. (2016) The cholinergic forebrain arousal system acts directly on the circadian pacemaker. Proc Natl Acad Sci U S A, 113, 13498-13503.

Ying, S.W. \& Rusak, B. (1994) Effects of serotonergic agonists on firing rates of photically responsive cells in the hamster suprachiasmatic nucleus. Brain Res, 651, 37-46.

Ying, S.W. \& Rusak, B. (1997) 5-HT 7 receptors mediate serotonergic effects on lightsensitive suprachiasmatic nucleus neurons. Brain Res, 755, 246-254.

This article is protected by copyright. All rights reserved. 


\section{Figure captions}

Figure 1 Average waveforms of wheel running activity during the last 6 days of baseline prior to implantation (solid lines) and the first 5 days after implantation (dashed lines) of the minipumps in saline-treated (top) and BMY7378-treated (middle) hamsters. Shading denotes the time of lights off (Zeitgeber time (ZT) 12-22). The bar graph denotes average activity levels (revolutions/minute) during the day, and the $1^{\text {st }}, 2^{\text {nd }}$, and $3^{\text {rd }}$ thirds of the night. * $p<0.05$, ** $p<0.01$. Individual data points in each group are denoted by the black circles.

Figure 2 Actograms from animals with either a saline-filled osmotic minipump (top left) or a BMY7378-filled minipump (top right). Every horizontal row depicts activity over a $24 \mathrm{~h}$ day, with subsequent days plotted below the previous days. Vertical marks in each row depict times during which the animal was engaged in wheel running, with the height of the marks being proportional to the amount of activity. Night is denoted by the shading. The time of implantation of the minipumps is denoted by the box containing an X. The brace brackets denote the range of days used for analysis. The bottom graph depicts the average phase angle of entrainments before and after implantation of the minipumps. Open circles over each bar denote the individual data points for each condition. The average $( \pm$ SEM) phase angles of entrainment were unaltered in saline (white bars) but were significantly advanced in animals treated with BMY7378 (shaded bars), both immediately after pump implantation and 10-14 days after pump

This article is protected by copyright. All rights reserved. 
implantation. Significant difference from the saline control and from baseline are denoted in the table on the right.

Figure 3 Actograms depicting phase shifts to phase delaying (left-hand plots) or phase advancing (right-hand plots) light pulses (white diamond) in animals with salinefilled (top row) or BMY7378-filled (bottom row) osmotic minipumps. The bar graph on the bottom presents the average ( \pm SEM) phase shifts in saline-treated (light grey) or BMY7378-treated (dark grey) animals. Individual data points contributing to each condition are depicted with open circles. Chronic BMY7378 treatment significantly attenuated phase advances to light. * $p<0.05$

Figure 4 Actograms depicting phase shifts to an injection of 8-OH-DPAT at CT8 (open diamond $\diamond$ ) in an animal with saline-filled (top) or BMY7378-filled (middle) osmotic minipumps. The bar graph on the bottom presents the average $( \pm$ SEM) phase shifts in saline-treated (white) or BMY7378-treated (grey) animals. Individual data points contributing to each condition are depicted with black circles. Chronic BMY7378 treatment significantly attenuated phase advances to 8-OH-DPAT. $* p<0.05$

Figure 5 qRT-PCR analysis of mRNA levels in the hypothalamus and prefrontal cortex for the $5-\mathrm{HT}_{1 \mathrm{~A}}$ receptor (top), the $5-\mathrm{HT}_{1 \mathrm{~B}}$ receptor (middle), and monoamine oxidase-A (MAO-A, bottom) at two circadian phases (circadian time (CT) 0 and CT12) in saline-treated (open bars) or BMY-7378-treated animals. Individual data points contributing to each condition are depicted with open circles. mRNA levels for the $5-\mathrm{HT}_{1 \mathrm{~A}}$ and the $\mathbf{5}-\mathrm{HT}_{1 \mathrm{~B}}$ receptors were significantly upregulated in the hypothalamus. ${ }^{*} p<0.05$ 
Figure 6 qRT-PCR analysis of mRNA levels in the brainstem for the 5-HT IA $_{\text {A }}$ receptor (top), the 5-HT $1 \mathrm{~B}$ receptor (middle), and monoamine oxidase-A (MAO-A, bottom) in saline-treated (open bars) or BMY-7378-treated animals.

Individual data points contributing to each condition are depicted with open circles. mRNA levels for the 5-HT ${ }_{1 \mathrm{~A}}$ receptor and for MAO-A were significantly downregulated in the brainstem. $* p<0.05$

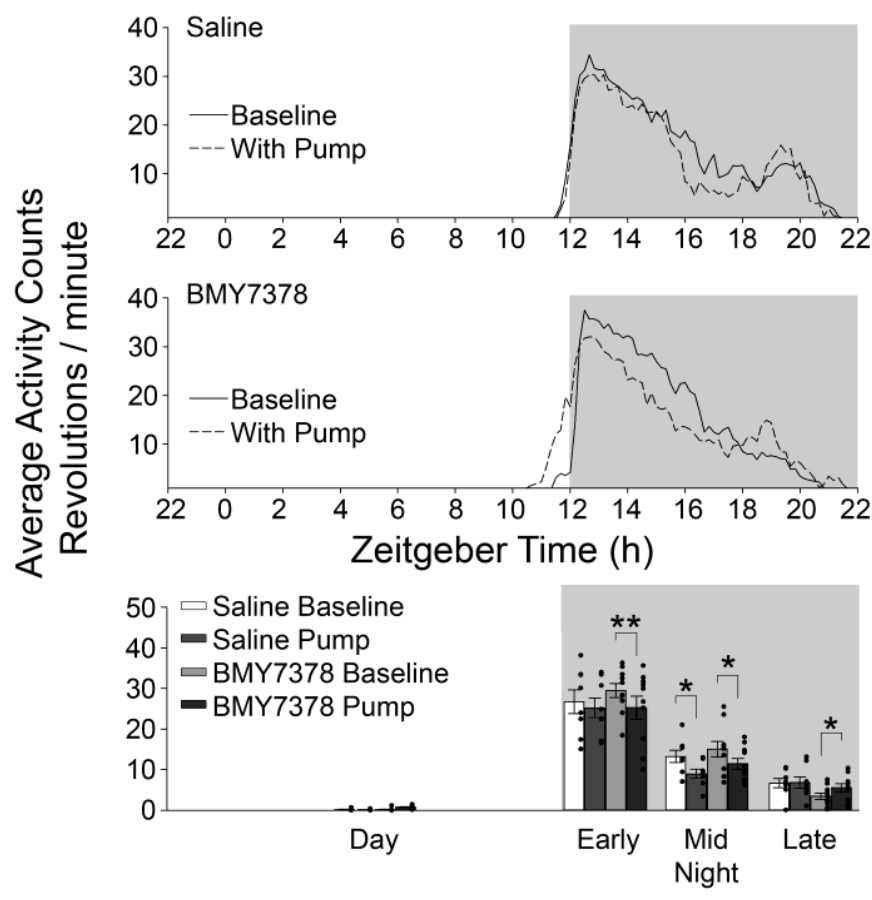

This article is protected by copyright. All rights reserved. 


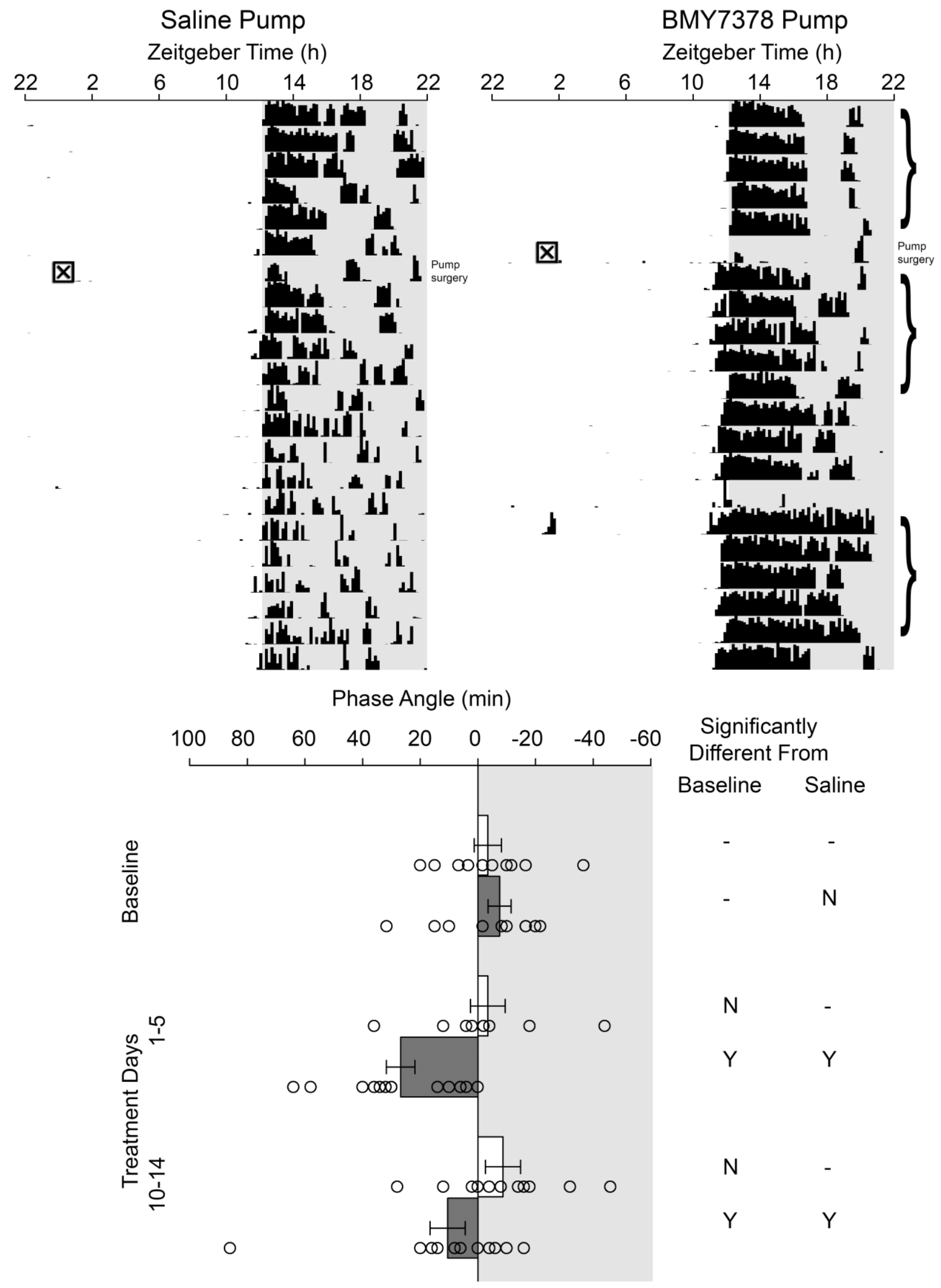

This article is protected by copyright. All rights reserved. 


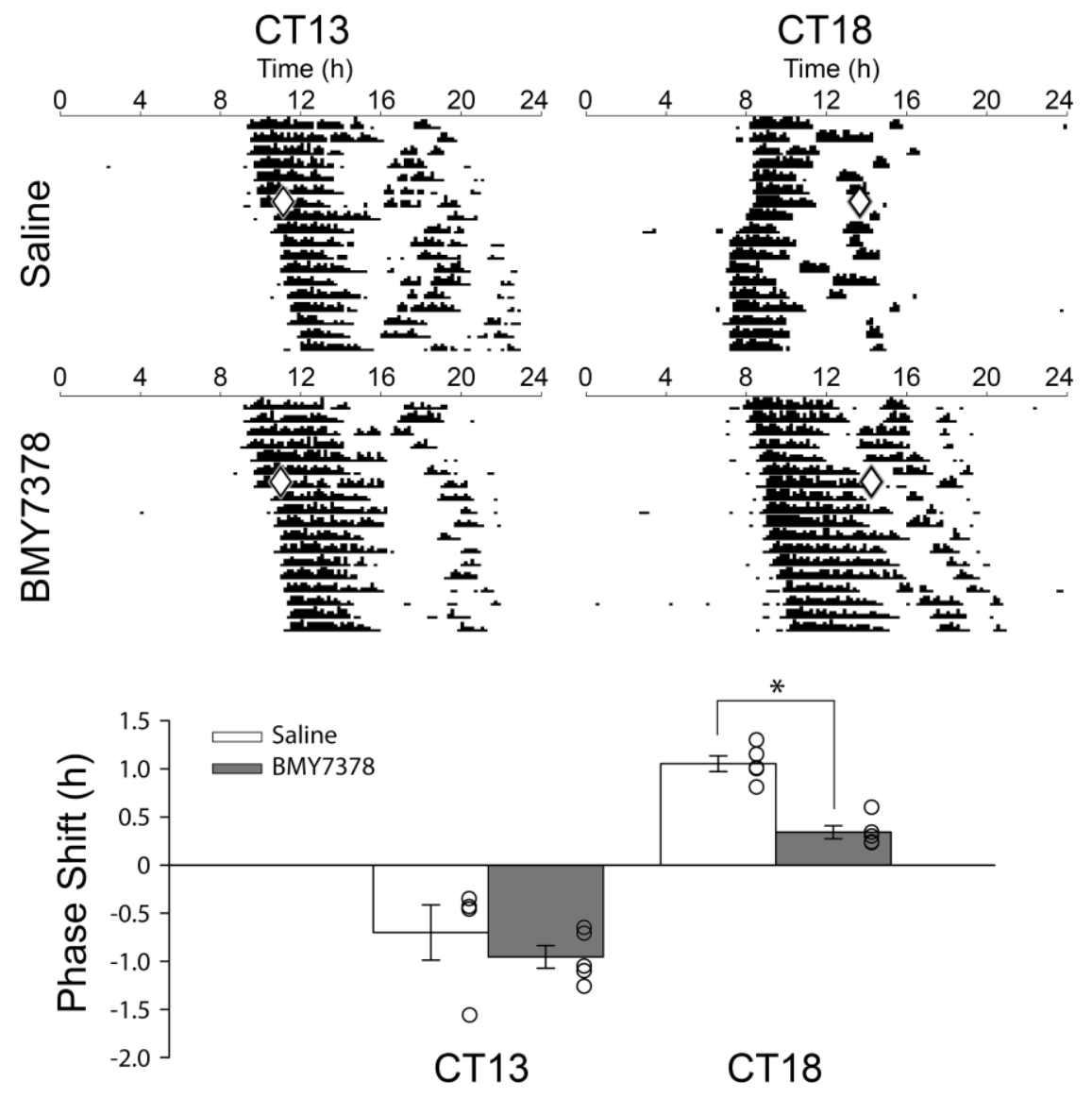

This article is protected by copyright. All rights reserved. 

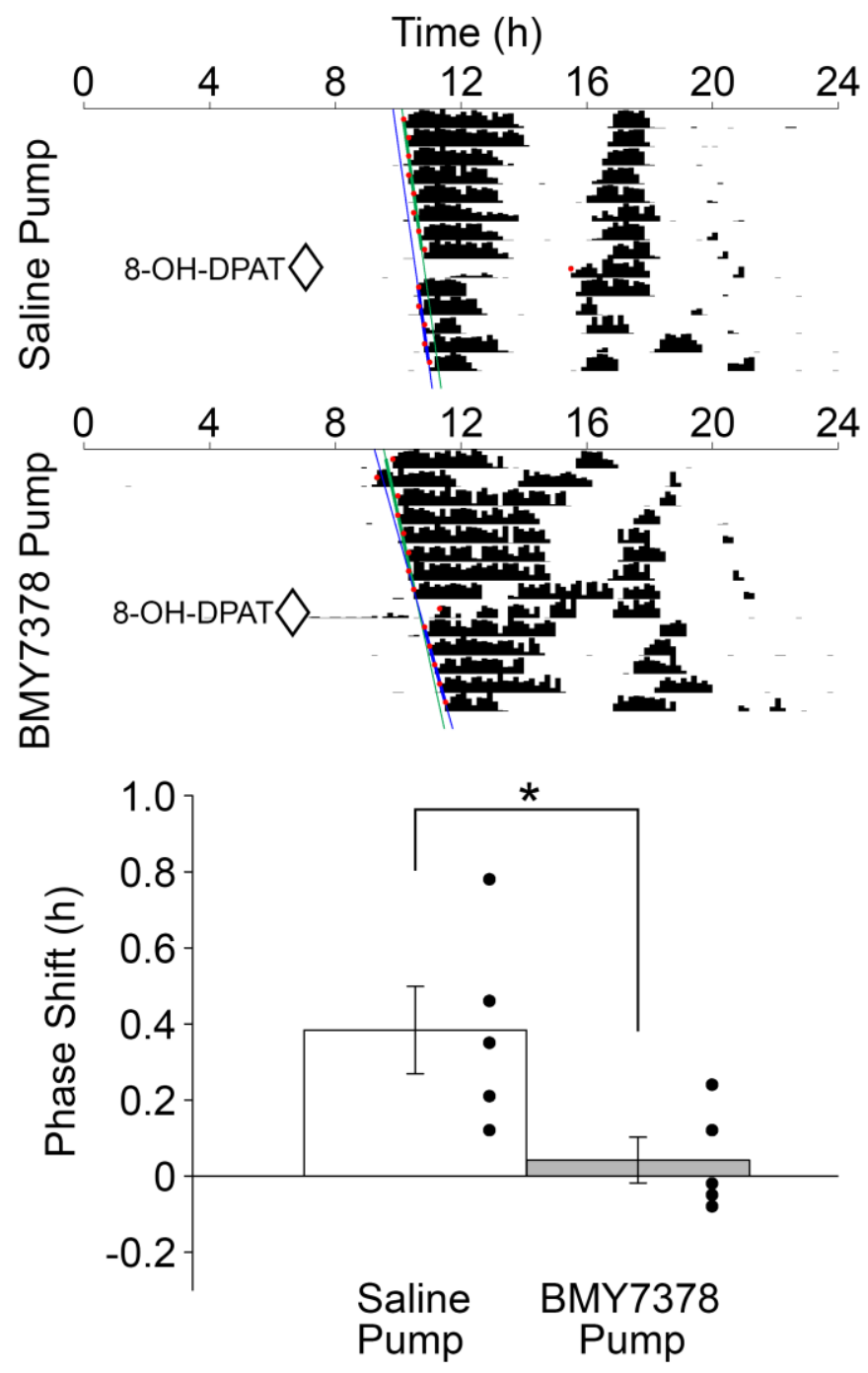

This article is protected by copyright. All rights reserved. 

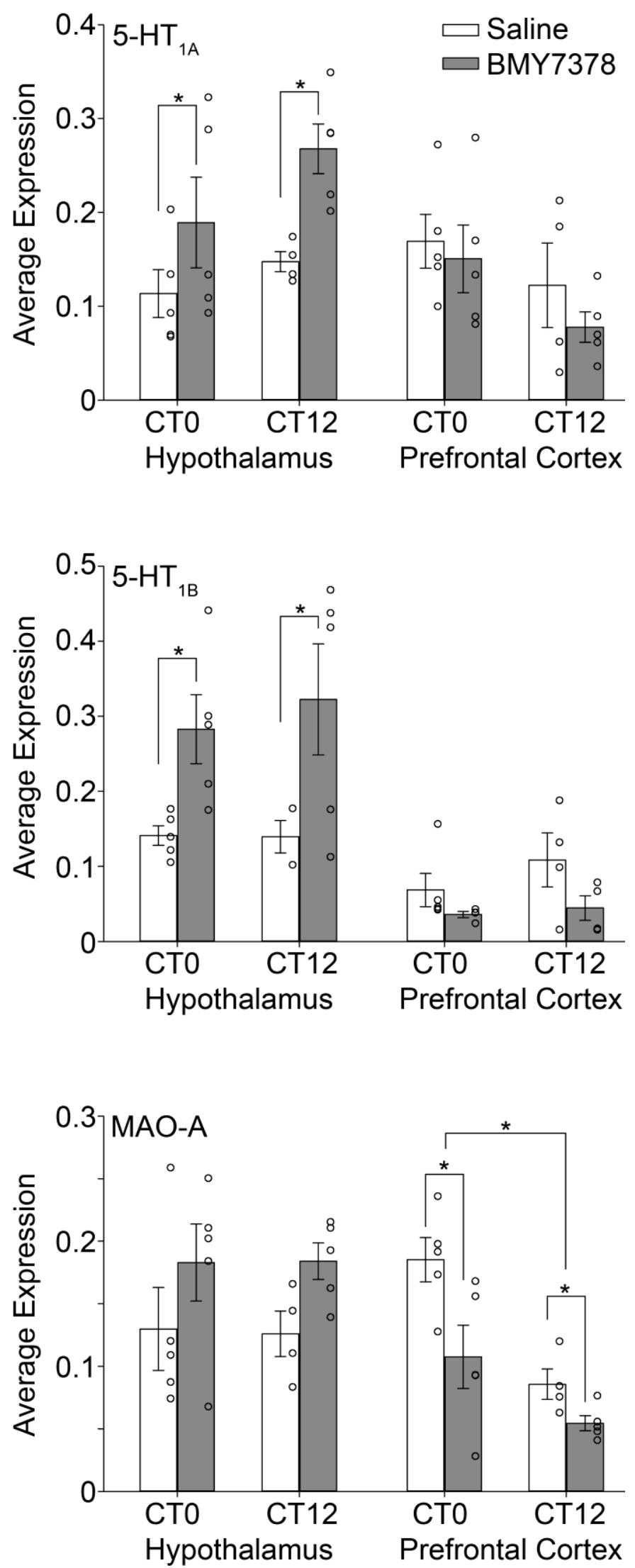

This article is protected by copyright. All rights reserved. 


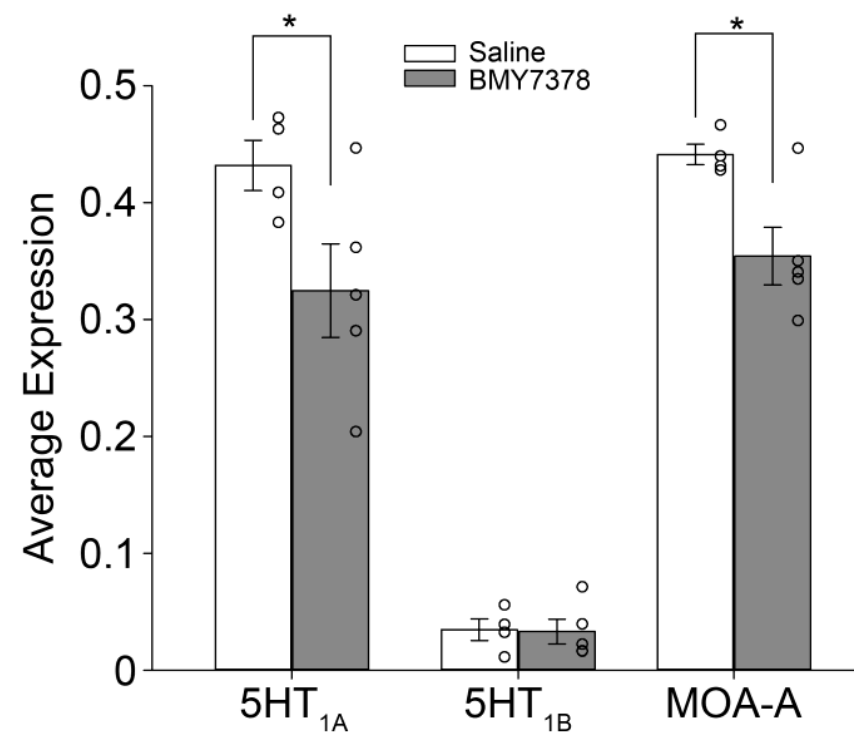

This article is protected by copyright. All rights reserved. 